\title{
UNIQUENESS THEOREMS FOR A CLASS OF GENERALIZED TRIGONOMETRICAL SERIES ${ }^{1}$
}

J. L. SMYRL

1. One of the applications which Cauchy made of the theory of residues was to the expansion of a function, $f(x)$, defined say in $(-\pi, \pi)$, as a series of the form

$$
a_{0}+\sum_{1}^{\infty}\left(a_{n} \cos \mu_{n} x+b_{n} \sin \mu_{n} x\right)
$$

where

$$
0=\mu_{0}<\mu_{1}<\mu_{2}<\cdots
$$

are the non-negative roots of the equation in $z$

$$
z+h \tan \pi z=0 \quad(h>0) .
$$

The formulae for the coefficients in (1) can be written

$$
a_{n}+i b_{n}=K_{n} \int_{-\pi}^{\pi} f(t) e^{i \mu_{n} t} d t \quad(n=1,2, \cdots)
$$

where

$$
K_{n}=\frac{2 \mu_{n}}{2 \pi \mu_{n}-\sin 2 \pi \mu_{n}}
$$

and

$$
a_{0}=K_{0} \int_{-\pi}^{\pi} f(t) d t
$$

where

$$
K_{0}=\frac{h}{2(1+\pi h)} .
$$

The modern treatment of the series (1) is due to Fejes [1]. We shall call (1) the F.A. series of $f(x)$ if equations (2) and (3) are satisfied.

In this note we are concerned with uniqueness theorems for convergent or summable series of the type (1). An important difference

Received by the editors March 1, 1954.

1 This note is a simplified account of part of a thesis which was awarded the Ph.D. degree at Queen's University, Belfast, in 1953. 
between the results obtained and those known for trigonometric series arises from the fact that there is a linear equation connecting the coefficients when (1) is an F.A. series. This equation (see Fejes [1]) is

$$
a_{0}=-\sum_{1}^{\infty} a_{n} \cos \mu_{n} \pi
$$

It is therefore desirable to enunciate our theorems in terms of a series without a constant term. We shall call

$$
\sum_{1}^{\infty}\left(a_{n} \cos \mu_{n} x+b_{n} \sin \mu_{n} x\right)
$$

the modified F.A. series (M.F.A. series) of $g(x)$ if there is a constant $a_{0}$ (which is then necessarily given by (4)) such that (1) is the F.A. series of $g(x)+a_{0}$. We shall then write

$$
g(x)+a_{0} \sim a_{0}+\sum_{1}^{\infty}\left(a_{n} \cos \mu_{n} x+b_{n} \sin \mu_{n} x\right) .
$$

We use the notation

$$
A_{n}(x)=a_{n} \cos \mu_{n} x+b_{n} \sin \mu_{n} x, \quad P(\boldsymbol{r}, x)=\sum A_{n}(x) \boldsymbol{r}^{n},
$$

and denote the upper or lower limits of $P(r, x)$, as $r \rightarrow 1-$, by $P^{*}(x)$, $P_{*}(x)$ respectively. We shall prove

TheOREM 1. If (5) converges in $(-\pi, \pi)$, except at an enumerable set, to a function $f(x) \in L(-\pi, \pi)$, then it is the M.F.A. series of $f(x)$.

THEOREM 2. If (i) $a_{n}=o(n), b_{n}=o(n)$, and

(ii) $P^{*}(x), P_{*}(x)$ are finite in $-\pi \leqq x \leqq \pi$ and integrable, then (5) is the sum of an M.F.A. series and of the series

$$
k \sum_{1}^{\infty} K_{n} \sin \mu_{n} \pi \sin \mu_{n} x,
$$

where $k$ is some constant.

Theorem 3. If, in Theorem $2, P^{*}(x)=P_{*}(x)=0$ almost everywhere, then $a_{n}=b_{n}=0$ for all $n$.

A more elaborate argument ${ }^{2}$ than is given in this note will prove that in Theorem 2 we may have an exceptional enumerable set at the points of which $P_{*}(x), P^{*}(x)$ are not required to be finite, but at which

2 This argument is given in my Ph.D. thesis. 


$$
\lim _{r \rightarrow 1}(1-r) P(r, x)=0 .
$$

2. We shall denote by $S_{n}(f ; x)=S_{n}(x)$ the $n$th partial sum of the F.A. series of $f(x)$. Corresponding to any integrable function $f(x)$ we define an associated function $\bar{f}(x)$, of period $4 \pi$, by the equations

$$
\bar{f}(x)=\left\{\begin{array}{lr}
f(x) & (-\pi \leqq x \leqq \pi), \\
-f(x-2 \pi) & (\pi<x<3 \pi) .
\end{array}\right.
$$

In this notation, the results of S. Verblunsky [2] concerning $S_{n}(f ; x)$ and its derivatives may be written

$$
\begin{aligned}
& S_{n}(f ; x)=S_{2 n}^{*}(\bar{f} ; x)+\epsilon_{n}^{(1)}(x) \\
& S_{n}^{\prime}(f ; x)=S_{2 n}^{* \prime}(\bar{f} ; x)-\frac{h}{2 \pi^{2}} \int_{-\pi}^{\pi} f(t) \frac{\xi \sin n \xi}{\sin \xi / 2} d t+\epsilon_{n}^{(2)}(x) \\
& S_{n}^{\prime \prime}(f ; x)=S_{2 n}^{* \prime \prime}(\bar{f} ; x)-\frac{h}{2 \pi^{2}} \int_{-\pi}^{\pi} f(t) \frac{d}{d \xi}\left(\frac{\xi \sin n \xi}{\sin \xi / 2}\right) d t+\epsilon_{n}^{(3)}(x)
\end{aligned}
$$

where $\epsilon_{n}^{(1)}(x) \rightarrow 0$ uniformly for $|x| \leqq \pi, \epsilon_{n}^{(2)}(x)$ and $\epsilon_{n}^{(3)}(x) \rightarrow 0$ for $|x| \leqq \pi, \xi=x-t$, and $S_{n}^{*}(f ; x)=S_{n}^{*}(x)$ denotes the $n$th partial sum of the Fourier series of $f(x)$.

Lemma 1. We have

$$
\lim _{r \rightarrow 1}(1-r) \sum S_{2 n}^{*}(\bar{f} ; x) r^{n}=\lim _{r \rightarrow 1}(1-r) \sum S_{n}^{*}(\bar{f} ; x) r^{n}
$$

when either side exists. Moreover, we may replace $S_{2 n}^{*}(x), S_{n}^{*}(x)$ by $S_{2 n}^{*^{\prime}}(x), S_{n}^{*^{\prime}}(x)$, or by $S_{2 n}^{* \prime \prime}(x), S_{n}^{* \prime \prime}(x)$, and in each of the results so obtained we may replace lim by lim sup or by lim inf throughout.

For

$$
\begin{aligned}
\lim _{r \rightarrow 1}(1-r) \sum S_{2 n}^{*}(x) r^{n} & =\lim _{r \rightarrow 1}\left(1-r^{2}\right) \sum S_{2 n}^{*}(x) r^{2 n} \\
& =2 \lim _{r \rightarrow 1}(1-r) \sum S_{2 n}^{*}(x) r^{2 n} \\
& =\lim _{r \rightarrow 1}(1-r) \sum S_{2 n}^{*}(x) r^{2 n} \\
& +\lim _{r \rightarrow 1} r(1-r) \sum S_{2 n-1}^{*}(x) r^{2 n-1}
\end{aligned}
$$

since, for a function of the type $\bar{f}(x)$, we have $S_{2 n}^{*}(x)=S_{2 n-1}^{*}(x)$. It follows that 


$$
\lim _{r \rightarrow 1}(1-r) \sum S_{2 n}^{*}(x) r^{n}=\lim _{r \rightarrow 1}(1-r) \sum S_{n}^{*}(x) r^{n}
$$

when either side exists. The other cases may be dealt with similarly.

3. We shall denote by $\mathcal{F}^{\prime}\{f(x)\}$ the series obtained by differentiating the F.A. series of $f(x)$, and by $\mathcal{F}^{\prime \prime}\{f(x)\}$ the series obtained by differentiating $\mathcal{F}^{\prime}\{f(x)\}$. The Poisson-sums of $\mathcal{F}^{\prime \prime}\{f(x)\}$ shall be denoted by $P(r, x), P^{*}(x), \mathscr{P}_{*}(x)$. We shall also use the notation

$$
\begin{aligned}
\Delta^{2} f(x, t) & =f(x+t)+f(x-t)-2 f(x), \\
D^{* 2} f(x) & =\limsup _{t \rightarrow 0} \frac{\Delta^{2} f(x, t)}{t^{2}}, \\
D_{*}^{2} f(x) & =\liminf _{t \rightarrow 0} \frac{\Delta^{2} f(x, t)}{t^{2}} .
\end{aligned}
$$

We may now prove

Lemma 2. If at a point $x_{0}\left(\left|x_{0}\right|<\pi\right)$

$$
\lim _{t \rightarrow 0} \frac{f\left(x_{0}+t\right)-f\left(x_{0}-t\right)}{2 t}=l
$$

where $l$ is not necessarily finite, then $\mathcal{F}^{\prime}\{f(x)\}$ is summable- $P$ at $x_{0}$ to $l$.

We consider the Poisson-limit of $S_{n}^{\prime}(f ; x)$. Since the second term on the right-hand side of (7) is $o(1)$ for $|x|<\pi$ the result follows from Lemma 1 and from the theory of Fourier series [3, p. 52] applied to the function $\bar{f}(x)$.

Lemma 3. If $f(-\pi+0)$ and $f(\pi-0)$ exist, and if

$$
\lim _{t \rightarrow 0} \frac{f(-\pi+t)+f(\pi-t)}{2 t}=l
$$

where $l$ is not necessarily finite, then $\mathcal{F}^{\prime}\{f(x)\}$ is summable-P at $x=\pi$ to $h f(-\pi+0)-l$, and at $x=-\pi$ to $l-h f(\pi-0)$.

We must reconsider the second term on the right-hand side of (7). At $x=\pi$ it equals $-(h / \pi) S_{2 n}^{*}(\bar{g} ; \pi)$, where $g(x)=(\pi-x) f(x)$, and so has the Poisson-limit

$$
-\frac{h}{2 \pi}[\bar{g}(\pi-0)+\bar{g}(\pi+0)]=h f(-\pi+0) .
$$

Using Lemma 1 and the theory of Fourier Series [3, p. 52], we find that the first term on the right-hand side of (7) has, at $x=\pi$, the 
Poisson-limit $-l$. Hence $\mathcal{F}^{\prime}\{f(x)\}$ is summable- $P$ at $x=\pi$ to $h f(-\pi+0)$ $-l$, and similarly at $x=-\pi$ to $l-h f(\pi-0)$.

Corollary 1. At $x=\pi, \mathcal{F}^{\prime}\{1\}$ is summable-P to $-\infty$, and at $x=-\pi$ to $\infty$.

Lemma 4. If $f(x)$ is continuous at $x=x_{0}\left(\left|x_{0}\right|<\pi\right)$, then

$$
D^{* 2} f\left(x_{0}\right) \geqq \Phi_{*}\left(x_{0}\right), \quad D_{*}^{2} f\left(x_{0}\right) \leqq \mathcal{P}^{*}\left(x_{0}\right) .
$$

Consider the second term on the right-hand side of (8). It equals

$$
\begin{gathered}
-\frac{h}{2 \pi^{2}} \int_{-\pi}^{\pi} f(t) \xi \frac{d}{d \xi}\left(\frac{\sin n \xi}{\sin \xi / 2}\right) d t-\frac{h}{2 \pi^{2}} \int_{-\pi}^{\pi} f(t) \frac{\sin n \xi}{\sin \xi / 2} d t \\
=-\frac{h}{\pi} S_{2 n}^{* \prime}(\bar{g} ; x)-\frac{h}{\pi} S_{2 n}^{*}(\bar{f} ; x)
\end{gathered}
$$

where $g(t)=(x-t) f(t)$. The second term in (9) has the Poisson-limit $-(h / \pi) f\left(x_{0}\right)$ at $x=x_{0}$. To see that the first term has equal but opposite Poisson-limit we note that

$$
\begin{aligned}
\lim _{t \rightarrow 0} \frac{\bar{g}\left(x_{0}+t\right)-\bar{g}\left(x_{0}-t\right)}{2 t} & =\lim _{t \rightarrow 0} \frac{-t f\left(x_{0}+t\right)-t f\left(x_{0}-t\right)}{2 t} \\
& =-f\left(x_{0}\right) .
\end{aligned}
$$

The lemma now follows from the theory of Fourier series [4, p. 268] applied, in conjunction with Lemma 1 , to the first term on the righthand side of (8).

4. Lemma 5. If (5) converges uniformly in $(-\pi, \pi)$ to $f(x) \in L(-\pi, \pi)$, then it is the M.F.A. series of $f(x)$.

By straightforward integration, remembering that $\mu_{n}+h \tan \pi \mu_{n}$ $=0$ for all $n$, we find that, if $n$ is nonzero,

$$
\int_{-\pi}^{\pi} \sin \mu_{m} t \sin \mu_{n} t d t= \begin{cases}0 & (m \neq n) \\ \frac{1}{K_{n}} & (m=n) .\end{cases}
$$

Hence

$$
\begin{aligned}
\int_{-\pi}^{\pi} \cos \mu_{m} t \cos \mu_{n} t d t & =\frac{2 \sin \mu_{n} \pi \cos \mu_{m} \pi}{\mu_{n}}+\frac{\mu_{m}}{\mu_{n}} \int_{-\pi}^{\pi} \sin \mu_{m} t \sin \mu_{n} t d t \\
& =\frac{2 \sin \mu_{n} \pi \cos \mu_{m} \pi}{\mu_{n}}+\theta_{m, n}
\end{aligned}
$$


where

$$
\theta_{m, n}= \begin{cases}0 & (m \neq n), \\ \frac{1}{K_{n}} & (m=n) .\end{cases}
$$

It then follows, by the term by term integration of a uniformly convergent series, that for any constant $c$

$$
\begin{aligned}
K_{n} \int_{-\pi}^{\pi}[f(t)+c] \cos \mu_{n} t d t= & \frac{2 c K_{n} \sin \mu_{n} \pi}{\mu_{n}} \\
& +\frac{2 K_{n} \sin \mu_{n} \pi}{\mu_{n}} \sum_{\nu=1}^{\infty} a_{\nu} \cos \mu_{\nu} \pi+a_{n} .
\end{aligned}
$$

Choosing $c=-\sum_{1}^{\infty} a_{n} \cos \mu_{n} \pi$, the right-hand side equals $a_{n}$. Further

$$
K_{n} \int_{-\pi}^{\pi}[f(t)+c] \sin \mu_{n} t d t=b_{n} .
$$

It follows that $f(x)+c \sim c+\sum_{1}^{\infty}\left(a_{n} \cos \mu_{n} x+b_{n} \sin \mu_{n} x\right)$, and the lemma is proved.

Proof of Theorem 1. The argument of the Cantor-Lebesgue Theorem [3, p. 267] applies to show that, since $A_{n}(x) \rightarrow 0$ p.p. in $(-\pi, \pi), a_{n}=o(1)$ and $b_{n}=o(1)$. Hence

$$
F^{*}(x)=-\sum_{1}^{\infty} A_{n}(x) / \mu_{n}^{2}
$$

converges uniformly in $(-\pi, \pi)$. If $F(x)=F^{*}(x)+c$, where $c=\sum_{1}^{\infty} a_{n} \cos \mu_{n} \pi / \mu_{n}^{2}$, we have, by Lemma 5 ,

$$
K_{n} \int_{-\pi}^{\pi} F(t) e^{i \mu_{n} t} d t=-\frac{a_{n}+i b_{n}}{\mu_{n}^{2}} \quad(n=1,2, \cdots) .
$$

Now $\mathcal{F}^{\prime \prime}\{F(x)\}$ is summable- $P$ to $f(x)$ and so, by Lemma 4 ,

$$
D^{* 2} F(x) \geqq f(x) \geqq D_{*}^{2} F(x)
$$

in $(-\pi, \pi)$ except in an enumerable set. Since $a_{n}=o(1), b_{n}=o(1)$, $F(x)$ is smooth. It follows $[3$, p. 276 , Theorem (iv)] that

$$
F(x)=\int_{-\pi}^{x} d y \int_{-\pi}^{y} f(t) d t+A x+B
$$

where $A, B$ are constants. We may now integrate by parts twice on the left-hand side of equation (10). Equating real and imaginary 
parts, and remembering that $F(\pi)+F(-\pi)=0$ by (4), we get

$$
\begin{aligned}
& a_{n}=K_{n} \int_{-\pi}^{\pi} f(t) \cos \mu_{n} t d t-K_{n} \cos \mu_{n} \pi \int_{-\pi}^{\pi} f(t) d t, \\
& b_{n}=K_{n} \int_{-\pi}^{\pi} f(t) \sin \mu_{n} t d t-\lambda K_{n} \sin \mu_{n} \pi,
\end{aligned}
$$

where $\lambda=F^{\prime}(\pi)+F^{\prime}(-\pi)+h[F(\pi)-F(-\pi)]$. Writing

$$
c^{\prime}=\frac{h}{2} \int_{-\pi}^{\pi} f(t) d t
$$

the equations (11) and (12) give

$$
\begin{aligned}
& K_{n} \int_{-\pi}^{\pi}\left[f(t)+c^{\prime}\right] \cos \mu_{n} t d t=a_{n}, \\
& K_{n} \int_{-\pi}^{\pi}\left[f(t)+c^{\prime}\right] \sin \mu_{n} t d t=b_{n}+\lambda K_{n} \sin \mu_{n} \pi .
\end{aligned}
$$

The left-hand side of (15) is $o(1)$, and we have seen that $b_{n}=o(1)$. Now $K_{n} \rightarrow 1 / \pi$ and

$$
\sin \mu_{n} \pi= \pm \frac{\mu_{n}}{\left(h^{2}+\mu_{n}^{2}\right)^{1 / 2}} .
$$

Hence $\lambda=0$. Lastly, (13) implies that

$$
c^{\prime}=K_{0} \int_{-\pi}^{\pi}\left[f(t)+c^{\prime}\right] d t .
$$

The theorem now follows from (14), (15), and (16).

5. Proof of Theorem 2. We have

$$
\sum_{1}^{\infty} \frac{A_{n}(x)}{n^{2}} \boldsymbol{r}^{n}=\int_{0}^{r} \frac{d \rho}{\rho} \int_{0}^{\rho} \frac{P(t, x)}{t} d t=I(\boldsymbol{r}) \quad \text { (say). }
$$

Now

$$
P(t, x) / t=\sum_{1}^{\infty} A_{n}(x) t^{n-1}
$$

which is a continuous function of $t$ in $0 \leqq t<1$. Since $P^{*}(x), P_{*}(x)$ are finite in $(-\pi, \pi), P(t, x) / t$ is bounded as $t \rightarrow 1$ and so $\lim _{r \rightarrow 1} I(r)$ exists. Hence $\lim _{r \rightarrow 1} \sum_{1}^{\infty} A_{n}(x) r^{n} / n^{2}$ exists for all $x$ in $(-\pi, \pi)$. It follows by Tauber's Theorem that $\sum A_{n}(x) / n^{2}$ is convergent for all $x$ in $(-\pi, \pi)$ 
and so therefore is $\sum A_{n}(x) / \mu_{n}^{2}$ since $\sum_{1}^{\infty}\left(A_{n}(x) / n^{2}-A_{n}(x) / \mu_{n}^{2}\right)$ is uniformly convergent. Thus $F^{*}(x)=-\sum_{1}^{\infty} A_{n}(x) / \mu_{n}^{2}$ is finite in $(-\pi, \pi)$ and can be written as $\sum_{1}^{\infty}\left(\alpha_{n} \cos \mu_{n} x+\beta_{n} \sin \mu_{n} x\right)\left(\alpha_{n}=o(1 / n)\right.$, $\left.\beta_{n}=o(1 / n)\right)$. Now, $\mu_{n}=n-1 / 2+O(1 / n)$, and so

$$
F^{*}(x)=\sum_{1}^{\infty}\left\{\alpha_{n} \cos (n-1 / 2) x+\beta_{n} \sin (n-1 / 2) x\right\}+G(x)
$$

where $G(x)$ is bounded. The series on the right-hand side is the sum, at $t=x / 2$, of the Fourier series of a function $g(t) \in L^{2}(-\pi, \pi)$. Since it converges for $t$ in $(-\pi / 2, \pi / 2)$ it must converge to $g(t)$ p.p. in $(-\pi / 2, \pi / 2)$. Hence $F^{*}(x)$ is integrable in $(-\pi, \pi)$, and by Theorem 1 the equation (10) is true with $F(x)=F^{*}(x)+c$. Now by the argument of Zygmund [3, p. 301] it can be shown that $F(x)$ is continuous. Hence $[3$, p. 276 , Theorem (iv) $]$ we have

$$
F(x)=\int_{-\pi}^{x} d y \int_{-\pi}^{y} f(t) d t+A x+B
$$

where $A, B$ are constants and $f(x)=\operatorname{Min}\left[D^{* 2} F(x), P^{*}(x)\right]$. Equations (14) and (15), with $c^{\prime}$ defined as in (13), may now be deduced from (10) exactly as in the proof of Theorem 1 . Writing $k=-\lambda$ we see that Theorem 2 is now proved.

6. Proof of Theorem 3. By Lemma $4, D^{* 2} F(x) \geqq 0$ and so $f(x)$ $=0$ p.p. in $(-\pi, \pi)$, where $F(x), f(x)$ are defined as in the proof of Theorem 2. Hence in this case Theorem 2 shows that (5) reduces to

$$
k \sum_{1}^{\infty} K_{n} \sin \mu_{n} \pi \sin \mu_{n} x .
$$

This series is $\mathcal{F}^{\prime}\{-k / 2\}$ which, by Corollary 1 , has infinite Poisson sum at $x=-\pi$ and $x=\pi$. Since $P^{*}(x), P_{*}(x)$ were supposed finite in $-\pi \leqq x \leqq \pi$ we must have $k=0$. The result follows.

I am indebted to Professor S. Verblunsky, under whose supervision this work was carried out, for his helpful criticism and advice.

\section{BIBLIOGRAPHY}

1. L. Fejes, Über die Fouriersche Reihe der Abkühlung, Acta Univ. Szeged. vol. 11 (1946-48) pp. 28-37.

2. S. Verblunsky, Sur une classe de séries exponentielles de Cauchy, Bull. Sci. Math. vol. 76 (1952) pp. 85-96.

3. A. Zygmund, Trigonometrical series, 1935.

4. A. Rajchman and A. Zygmund, Math. Zeit. vol. 25 (1926) pp. 261-273 (268).

The Queen's University, Belfast 